\title{
Oscillatory behavior of a second order nonlinear advanced differential equation with mixed neutral terms
}

\author{
Hongwei Shi ${ }^{1}$ and Yuzhen Bai ${ }^{*}$ (D)
}

"Correspondence:
baiyu99@126.com
${ }^{1}$ School of Mathematical Sciences,
Qufu Normal University, Qufu,
P.R. China

P.R. China

\begin{abstract}
In this paper, we present several new oscillation criteria for a second order nonlinear differential equation with mixed neutral terms of the form

$$
\left(r(t)\left(z^{\prime}(t)\right)^{\alpha}\right)^{\prime}+q(t) x^{\beta}(\sigma(t))=0, \quad t \geq t_{0}
$$

where $z(t)=x(t)+p_{1}(t) x(\tau(t))+p_{2}(t) x(\lambda(t))$ and $\alpha, \beta$ are ratios of two positive odd integers. Our results improve and complement some well-known results which were published recently in the literature. Two examples are given to illustrate the efficiency of our results.
\end{abstract}

MSC: 34K06; 34K11

Keywords: Oscillation; Second order; Mixed neutral terms; Advanced differential equation

\section{Introduction}

In the article, we consider the oscillatory and asymptotic behavior of solutions to a second order nonlinear advanced differential equation with mixed neutral terms of the form

$$
\left(r(t)\left(z^{\prime}(t)\right)^{\alpha}\right)^{\prime}+q(t) x^{\beta}(\sigma(t))=0, \quad t \geq t_{0}
$$

where $z(t)=x(t)+p_{1}(t) x(\tau(t))+p_{2}(t) x(\lambda(t))$. We assume the following conditions hold throughout this paper.

(H1) $\alpha$ and $\beta$ are ratios of two positive odd integers;

(H2) $r, \sigma \in C^{1}\left(\left[t_{0}, \infty\right),(0, \infty)\right), r(t)>0, \sigma(t) \geq t, \sigma^{\prime}(t) \geq 0, \lim _{t \rightarrow \infty} \sigma(t)=\infty$;

(H3) $\tau, \lambda \in C\left(\left[t_{0}, \infty\right), R\right), \tau(t) \leq t, \lambda(t) \geq t, \lim _{t \rightarrow \infty} \tau(t)=\lim _{t \rightarrow \infty} \lambda(t)=\infty$;

(H4) $p_{1}, p_{2} \in C\left(\left[t_{0}, \infty\right),[0,1)\right), q \in C\left(\left[t_{0}, \infty\right),[0, \infty)\right), q(t)$ is not identically zero in any interval of $\left[t_{0}, \infty\right)$.

By a solution of Eq. (1.1) we mean a function $x \in C\left[T_{x}, \infty\right), T_{x} \geq T_{0}$, which has the property $r(t)\left(z^{\prime}(t)\right)^{\alpha} \in C^{1}\left(\left[T_{x}, \infty\right), R\right)$ and satisfies $(1.1)$ on $\left[T_{x}, \infty\right)$. In this paper we only consider the nontrivial solution of Eq. (1.1) which satisfies $\sup \{|x(t)|: t \geq T\}>0$ for all $T \geq T_{x}$. A solution of (1.1) is called oscillatory if it has arbitrarily large zeros on $\left[T_{x}, \infty\right)$;

(c) The Author(s) 2019. This article is distributed under the terms of the Creative Commons Attribution 4.0 International License (http://creativecommons.org/licenses/by/4.0/), which permits unrestricted use, distribution, and reproduction in any medium, provided you give appropriate credit to the original author(s) and the source, provide a link to the Creative Commons license, and indicate if changes were made. 
Otherwise, it is said to be nonoscillatory. Equation (1.1) is said to be oscillatory if all its solutions oscillate.

Following Trench [21], we shall say that Eq. (1.1) is in canonical form if

$$
\int_{t_{0}}^{\infty} r^{-\frac{1}{\alpha}}(s) d s=\infty
$$

Conversely, we say that (1.1) is in noncanonical form if

$$
\int_{t_{0}}^{\infty} r^{-\frac{1}{\alpha}}(s) d s<\infty
$$

Advanced differential equations can find applications in a mass of real world problems where the evolution rate depends on present and future values of the quantity. Therefore, taking into account the impact of potential future actions, an advance could be introduced into the equation, which is available at the present and beneficial in the process of decision making. For instance, we can find numerous applications in mechanical control engineering, economical problems, population dynamics, neural networks and the field of time symmetric electrodynamics; see [14].

The establishment of oscillatory and/or nonoscillatory criteria for differential equations with deviating arguments, which was first studied by Fite [15] in 1921, has always been a very active research field. Several reviews and references of known results can be found in the monographs [3-6]. Up to now, most literature has been devoted to the study of delay differential equations, but few studies have considered the equations with advanced arguments. Therefore, recent studies have attempted to improve the already existing oscillation criteria.

Džurina [12] studied the advanced canonical equation of the form

$$
\left(r(t) y^{\prime}(t)\right)^{\prime}+q(t) y(\sigma(t))=0
$$

and established a new comparison principle by using new monotonic properties of nonoscillatory solutions and iterated exponentiation. Agarwal et al. [7] used an approach that leads to two independent conditions, eliminating increasing and decreasing positive solutions, respectively. Baculíková [9] and Jadlovská [17] investigated the second order linear advanced equation

$$
y^{\prime \prime}(t)+q(t) y(\sigma(t))=0
$$

and gave new oscillation results employing some iterative techniques. Recently, Chatzarakis et al. [10] investigated the second order half-linear differential equation with advanced argument

$$
\left(r(t)\left(y^{\prime}(t)\right)^{\alpha}\right)^{\prime}+q(t) y^{\alpha}(\sigma(t))=0
$$

and established new oscillation criteria under the condition (1.3).

Motivated by the above work, we will consider a generalized nonlinear advanced differential equations with mixed neutral terms and establish new sufficient conditions for 
oscillation of Eq. (1.1) under the condition (1.3). Our results presented in Sect. 2 improve and complement those of Refs. [1, 2, 7-10, 12, 16, 17, 19, 20,23]. Two examples are addressed to illustrate the efficiency of the main results in Sect. 3 and the conclusions are given in Sect. 4.

\section{Main results}

In this section, we present some lemmas and our new sufficient conditions for oscillation of Eq. (1.1). For the sake of convenience, we use the following notation:

$$
\begin{array}{ll}
R(t)=\int_{t_{0}}^{t} r^{-\frac{1}{\alpha}}(s) d s, & Q_{1}(t)=1-p_{1}(\sigma(t))-p_{2}(\sigma(t)) \frac{R(\lambda(\sigma(t)))}{R(\sigma(t))}, \\
\pi(t)=\int_{t}^{\infty} r^{-\frac{1}{\alpha}}(s) d s, & Q_{2}(t)=1-p_{1}(\sigma(t)) \frac{\pi(\tau(\sigma(t)))}{\pi(\sigma(t))}-p_{2}(\sigma(t)),
\end{array}
$$

where $t \in\left[t_{0}, \infty\right)$.

In what follows we need only to consider the eventually positive solutions of Eq. (1.1), since if $x$ satisfies Eq. (1.1), then $-x$ is also its solution. Without loss of generality, we only give proofs for the positive solutions. We begin with the following lemmas.

Lemma 2.1 If $x(t)$ is an eventually positive solution of equation (1.1), then the corresponding function $z(t)$ satisfies one of two cases eventually:

Case 1. $z(t)>0, r(t)\left(z^{\prime}(t)\right)^{\alpha}>0$ and $\left(r(t)\left(z^{\prime}(t)\right)^{\alpha}\right)^{\prime} \leq 0$;

Case 2. $z(t)>0, r(t)\left(z^{\prime}(t)\right)^{\alpha}<0$ and $\left(r(t)\left(z^{\prime}(t)\right)^{\alpha}\right)^{\prime} \leq 0$.

Proof Suppose that $x(t)$ is an eventually positive solution of equation (1.1). In view of (H3) and (H4), there exists $t_{1} \geq t_{0}$ such that $x(\tau(t))>0, x(\sigma(t))>0, x(\lambda(t))>0$ for all $t \geq t_{1}$, then $z(t)=x(t)+p(t) x(\tau(t))+x(\lambda(t)) \geq x(t)>0$, for all $t \geq t_{1}$. From Eq. (1.1) we have

$$
\left(r(t)\left(z^{\prime}(t)\right)^{\alpha}\right)^{\prime}=-q(t) x^{\beta}(\sigma(t)) \leq 0, \quad t \geq t_{1}
$$

which means that $r(t)\left(z^{\prime}(t)\right)^{\alpha}$ is nonincreasing for all $t \geq t_{1}$. Then $r(t)\left(z^{\prime}(t)\right)^{\alpha}>0$ or $r(t)\left(z^{\prime}(t)\right)^{\alpha}<0$, and the proof is complete.

Lemma 2.2 If $x(t)$ is a positive solution of equation (1.1) satisfying Case 1 of Lemma 2.1, then

$$
z(t) \geq R(t) r^{\frac{1}{\alpha}}(t) z^{\prime}(t)
$$

and $\frac{z(t)}{R(t)}$ is nonincreasing for all $t \geq t_{1}$. Furthermore,

$$
x(t) \geq Q_{1}(t) z(t)
$$

on $t \in\left[t_{1}, \infty\right)$.

Proof From Case 1, $z(t)>0, z^{\prime}(t)>0$. Combining condition (1.2), we see that

$$
z(t)=z\left(t_{1}\right)+\int_{t_{1}}^{t} z^{\prime}(s) d s \geq \int_{t_{1}}^{t} \frac{r^{\frac{1}{\alpha}}(s) z^{\prime}(s)}{r^{\frac{1}{\alpha}}(s)} d s \geq R(t) r^{\frac{1}{\alpha}}(t) z^{\prime}(t)
$$


and

$$
\left(\frac{z(t)}{R(t)}\right)^{\prime}=\frac{z^{\prime}(t) R(t)-R^{\prime}(t) z(t)}{R^{2}(t)}=-\frac{z(t)-R(t) r^{\frac{1}{\alpha}}(t) z^{\prime}(t)}{r^{\frac{1}{\alpha}}(t) R^{2}(t)} \leq 0 .
$$

Using the monotonicity of $z(t)$ and $\frac{z(t)}{R(t)}$, we have

$$
\begin{aligned}
x(t) & =z(t)-p_{1}(t) x(\tau(t))-p_{2}(t) x(\lambda(t)) \geq z(t)-p_{1}(t) z(\tau(t))-p_{2}(t) z(\lambda(t)) \\
& \geq\left(1-p_{1}(t)-p_{2}(t) \frac{R(\lambda(t))}{R(t)}\right) z(t)=Q_{1}(t) z(t) .
\end{aligned}
$$

Lemma 2.3 If $x(t)$ is a positive solution of Eq. (1.1) satisfying Case 2 of Lemma 2.1, then

$$
z(t) \geq-\pi(t) r^{\frac{1}{\alpha}}(t) z^{\prime}(t)
$$

and $\frac{z(t)}{\pi(t)}$ is nondecreasing for all $t \geq t_{1}$. Furthermore,

$$
x(t) \geq Q_{2}(t) z(t)
$$

on $t \in\left[t_{1}, \infty\right)$.

Proof From Case 2, $z(t)>0, z^{\prime}(t)<0$. Using condition (1.3), we have

$$
z(l)=z(t)+\int_{t}^{l} z^{\prime}(s) d s=z(t)+\int_{t}^{l} \frac{r^{\frac{1}{\alpha}}(s) z^{\prime}(s)}{r^{\frac{1}{\alpha}}(s)} d s \leq z(t)+r^{\frac{1}{\alpha}}(t) z^{\prime}(t) \int_{t}^{l} r^{-\frac{1}{\alpha}}(s) d s .
$$

Letting $l \rightarrow \infty$, we get

$$
0 \leq z(t)+\pi(t) r^{\frac{1}{\alpha}}(t) z^{\prime}(t)
$$

Then

$$
z(t) \geq-\pi(t) r^{\frac{1}{\alpha}}(t) z^{\prime}(t)
$$

hence

$$
\left(\frac{z(t)}{\pi(t)}\right)^{\prime}=\frac{z^{\prime}(t) \pi(t)-\pi^{\prime}(t) z(t)}{\pi^{2}(t)}=\frac{z(t)+\pi(t) r^{\frac{1}{\alpha}}(t) z^{\prime}(t)}{r^{\frac{1}{\alpha}}(t) \pi^{2}(t)} \geq 0 .
$$

Using the monotonicity of $z(t)$ and $\frac{z(t)}{\pi(t)}$, we have

$$
\begin{aligned}
x(t) & =z(t)-p_{1}(t) x(\tau(t))-p_{2}(t) x(\lambda(t)) \geq z(t)-p_{1}(t) z(\tau(t))-p_{2}(t) z(\lambda(t)) \\
& \geq\left(1-p_{1}(t) \frac{\pi(\tau(t))}{\pi(t)}-p_{2}(t)\right) z(t)=Q_{2}(t) z(t) .
\end{aligned}
$$


Lemma 2.4 Assume that (1.3) holds and

$$
\int_{t_{0}}^{\infty} q(s) Q_{1}^{\beta}(\sigma(s)) d s=\infty
$$

Suppose that $x(t)$ is a positive solution of Eq. (1.1) on $\left[t_{1}, \infty\right)$, where $t_{1} \in\left[t_{0}, \infty\right)$ is sufficiently large, then Case 2 of Lemma 2.1 holds.

Proof Suppose that $x(t)$ is a positive solution of equation (1.1) on $t \in\left[t_{1}, \infty\right)$. From Lemma 2.1, we have Case 1 and Case 2. If Case 1 holds, then there exists $t_{2} \geq t_{1}$ such that $z^{\prime}(t)>0$ on $\left[t_{2}, \infty\right)$. Combining (1.1) and equation (2.2), we get

$$
\left(r(t)\left(z^{\prime}(t)\right)^{\alpha}\right)^{\prime} \leq-q(t) Q_{1}^{\beta}(\sigma(t)) z^{\beta}(\sigma(t)), \quad t \geq t_{1} .
$$

Define the function $w$ by

$$
w(t):=\frac{r(t)\left(z^{\prime}(t)\right)^{\alpha}}{z^{\beta}(\sigma(t))}>0, \quad t \geq t_{1} .
$$

Differentiating the above formula, we have

$$
w^{\prime}(t) \leq-q(t) Q_{1}^{\beta}(\sigma(t))-\frac{\beta w(t) z^{\prime}(\sigma(t)) \sigma^{\prime}(t)}{z(\sigma(t))} \leq-q(t) Q_{1}^{\beta}(\sigma(t)) .
$$

Integrating both sides of (2.8) from $t_{2}$ to $t$ and using (2.6), we obtain

$$
w(t) \leq w\left(t_{2}\right)-\int_{t_{2}}^{t} q(s) Q_{1}^{\beta}(\sigma(s)) d s \rightarrow-\infty, \quad \text { as } t \rightarrow \infty,
$$

which contradicts the fact $w(t)>0$. Thus, Case 1 is impossible and $z$ satisfies Case 2 for $t \geq t_{1}$. The proof is complete.

Theorem 2.5 Let $\alpha \geq \beta$. Assume that (1.3), (2.6) and

$$
\int_{t_{0}}^{\infty}\left(\frac{1}{r(t)} \int_{t_{0}}^{t} q(s) Q_{2}^{\beta}(\sigma(s)) d s\right)^{1 / \alpha} d t=\infty
$$

hold. Suppose that Eq. (1.1) has a positive solution $x(t)$ on $\left[t_{1}, \infty\right)$. Then $z(t)$ satisfies Case 2 on $\left[t_{1}, \infty\right)$ and

$$
\lim _{t \rightarrow \infty} x(t)=0 .
$$

Moreover, there exist positive constants $C_{1}$ and $C_{2}$ and a real number $t_{*} \in\left[t_{1}, \infty\right)$ such that

$$
C_{1} Q_{2}(t) \pi(t) \leq x(t) \leq C_{2} \exp \left(-\int_{t_{0}}^{t} \frac{\pi(\sigma(s))\left(\int_{t_{0}}^{s} q(u) Q_{2}^{\beta}(\sigma(u)) d u\right)^{\frac{1}{\alpha}}}{\pi(s) r^{1 / \alpha}(s)} d s\right)
$$

on $t \in\left[t_{*}, \infty\right)$. 
Proof Suppose that $x(t)$ is a positive solution of Eq. (1.1) on $\left[t_{1}, \infty\right)$. From Lemma 2.4, we see that $z(t)$ satisfies Case 2 for $t \geq t_{1}$.

Since $z(t)$ is nonincreasing and $z(t)>0$, there exists a constant $c \geq 0$ such that $\lim _{t \rightarrow \infty} x(t)=c \geq 0$. We now claim that $c=0$. If not, assume that $c>0$, combining (1.1), we have

$$
-\left(r(t)\left(z^{\prime}(t)\right)^{\alpha}\right)^{\prime}=q(t) x^{\beta}(\sigma(t)), \quad t \geq t_{0} .
$$

Integrating the above inequality from $t_{1}$ to $t$, we get

$$
r(t)\left(z^{\prime}(t)\right)^{\alpha}-r\left(t_{1}\right)\left(z^{\prime}\left(t_{1}\right)\right)^{\alpha}=-\int_{t_{1}}^{t} q(s) x^{\beta}(\sigma(s)) d s, \quad t \geq t_{1},
$$

which implies that

$$
r(t)\left(z^{\prime}(t)\right)^{\alpha} \leq-\int_{t_{1}}^{t} q(s) x^{\beta}(\sigma(s)) d s, \quad t \geq t_{1}
$$

then

$$
z^{\prime}(t) \leq-\left(\frac{1}{r(t)} \int_{t_{1}}^{t} q(s) x^{\beta}(\sigma(s)) d s\right)^{\frac{1}{\alpha}}, \quad t \geq t_{1}
$$

Integrating (2.12) from $t_{1}$ to $t$, we obtain

$$
z(t)-z\left(t_{1}\right) \leq-\int_{t_{1}}^{t}\left(\frac{1}{r(s)} \int_{t_{1}}^{s} q(u) x^{\beta}(\sigma(u)) d u\right)^{\frac{1}{\alpha}} d s, \quad t \geq t_{1}
$$

From Lemma 2.3, we have

$$
\begin{aligned}
z(t)-z\left(t_{1}\right) & \leq-\int_{t_{1}}^{t}\left(\frac{1}{r(s)} \int_{t_{1}}^{s} q(u) Q_{2}^{\beta}(\sigma(u)) z^{\beta}(\sigma(u)) d u\right)^{\frac{1}{\alpha}} d s \\
& \leq-z^{\frac{\beta}{\alpha}}(\sigma(t)) \int_{t_{1}}^{t}\left(\frac{1}{r(s)} \int_{t_{1}}^{s} q(u) Q_{2}^{\beta}(\sigma(u)) d u\right)^{\frac{1}{\alpha}} d s \\
& \leq-x^{\frac{\beta}{\alpha}}(\sigma(t)) \int_{t_{1}}^{t}\left(\frac{1}{r(s)} \int_{t_{1}}^{s} q(u) Q_{2}^{\beta}(\sigma(u)) d u\right)^{\frac{1}{\alpha}} d s .
\end{aligned}
$$

Letting $t \rightarrow \infty$ in the above inequality, we see that $z(t) \rightarrow-\infty$ as $t \rightarrow \infty$ which is a contradiction. Hence, $c=0$.

Next, we prove that inequality (2.11) holds. From Lemma 2.3, we see that $\frac{z(t)}{\pi(t)}$ is nondecreasing for all $t \geq t_{1}$. Thus, there exist $C_{1}>0$ and $t_{2}>t_{1}$ such that

$$
z(t) \geq C_{1} \pi(t), \quad t \geq t_{2}
$$

Using (2.4), we get

$$
x(t) \geq Q_{2}(t) z(t) \geq C_{1} Q_{2}(t) \pi(t) .
$$


Integrating (1.1) from $t_{2}$ to $t$, we have

$$
\begin{aligned}
& -r(t)\left(z^{\prime}(t)\right)^{\alpha} \\
& \quad=-r\left(t_{2}\right)\left(z^{\prime}\left(t_{2}\right)\right)^{\alpha}+\int_{t_{2}}^{t} q(s) x^{\beta}(\sigma(s)) d s \\
& \quad \geq-r\left(t_{2}\right)\left(z^{\prime}\left(t_{2}\right)\right)^{\alpha}+z^{\beta}(\sigma(t)) \int_{t_{2}}^{t} q(s) Q_{2}^{\beta}(\sigma(s)) d s \\
& \quad \geq-r\left(t_{2}\right)\left(z^{\prime}\left(t_{2}\right)\right)^{\alpha}+z^{\beta}(\sigma(t)) \int_{t_{0}}^{t} q(s) Q_{2}^{\beta}(\sigma(s)) d s-z^{\beta}(\sigma(t)) \int_{t_{0}}^{t_{2}} q(s) Q_{2}^{\beta}(\sigma(s)) d s .
\end{aligned}
$$

In view of (2.10), there exists $t_{3}>t_{2}$ such that

$$
-r\left(t_{3}\right)\left(z^{\prime}\left(t_{3}\right)\right)^{\alpha}-z^{\beta}(\sigma(t)) \int_{t_{0}}^{t_{3}} q(s) Q_{2}^{\beta}(\sigma(s)) d s>0, \quad t \geq t_{3} .
$$

Therefore,

$$
-r(t)\left(z^{\prime}(t)\right)^{\alpha} \geq z^{\beta}(\sigma(t)) \int_{t_{0}}^{t} q(s) Q_{2}^{\beta}(\sigma(s)) d s, \quad t \geq t_{3} .
$$

Using Lemma 2.3 in the above inequality, we find

$$
\begin{aligned}
-r(t)\left(z^{\prime}(t)\right)^{\alpha} & \geq \frac{z^{\beta-\alpha}(\sigma(t)) z^{\alpha}(\sigma(t))}{\pi^{\alpha}(\sigma(t))} \pi^{\alpha}(\sigma(t)) \int_{t_{0}}^{t} q(s) Q_{2}^{\beta}(\sigma(s)) d s \\
& \geq m_{1} \frac{z^{\alpha}(t)}{\pi^{\alpha}(t)} \pi^{\alpha}(\sigma(t)) \int_{t_{0}}^{t} q(s) Q_{2}^{\beta}(\sigma(s)) d s,
\end{aligned}
$$

where $m_{1}>0$ is a constant and $z^{\beta-\alpha}(\sigma(t)) \geq m_{1}$ for $t \geq t_{3}$, which implies that

$$
\frac{z^{\prime}(t)}{z(t)} \leq-\frac{m_{1} \pi(\sigma(t))}{\pi(t) r^{1 / \alpha}(t)}\left(\int_{t_{0}}^{t} q(s) Q_{2}^{\beta}(\sigma(s)) d s\right)^{\frac{1}{\alpha}}
$$

Integrating (2.15) from $t_{3}$ to $t$, we get

$$
\begin{aligned}
x(t) \leq z(t) & \leq z\left(t_{3}\right) \exp \left(-\int_{t_{3}}^{t} \frac{m_{1} \pi(\sigma(u))}{\pi(u) r^{1 / \alpha}(u)}\left(\int_{t_{0}}^{u} q(s) Q_{2}^{\beta}(\sigma(s)) d s\right)^{\frac{1}{\alpha}} d u\right) \\
& =C_{2} \exp \left(-\int_{t_{0}}^{t} \frac{m_{1} \pi(\sigma(u))}{\pi(u) r^{1 / \alpha}(u)}\left(\int_{t_{0}}^{u} q(s) Q_{2}^{\beta}(\sigma(s)) d s\right)^{\frac{1}{\alpha}} d u\right),
\end{aligned}
$$

where

$$
C_{2}:=z\left(t_{3}\right) \exp \left(-\int_{t_{0}}^{t_{3}} \frac{m_{1} \pi(\sigma(u))}{\pi(u) r^{1 / \alpha}(u)}\left(\int_{t_{0}}^{u} q(s) Q_{2}^{\beta}(\sigma(s)) d s\right)^{\frac{1}{\alpha}} d u\right)>0 .
$$

The proof is complete. 
Theorem 2.6 Assume that (1.3) and (2.6) hold. If

$$
\int_{t_{0}}^{\infty}\left(\frac{1}{r(t)} \int_{t_{0}}^{t} q(s) Q_{2}^{\beta}(\sigma(s)) \pi^{\beta}(\sigma(s)) d s\right)^{\frac{1}{\alpha}} d t=\infty
$$

then Eq. (1.1) is oscillatory.

Proof Suppose that $x(t)$ is a positive solution of equation (1.1) on $\left[t_{1}, \infty\right)$. From Lemma 2.4, we see that $z$ satisfies Case 2 for $t \geq t_{1}$.

From (1.1), (2.4) and (2.14), we obtain

$$
\begin{aligned}
-\left(r(t)\left(z^{\prime}(t)\right)^{\alpha}\right)^{\prime} & =q(t) x^{\beta}(\sigma(t)) \\
& \geq q(t) Q_{2}^{\beta}(\sigma(t)) z^{\beta}(\sigma(t)) \\
& \geq C_{1}^{\beta} q(t) Q_{2}^{\beta}(\sigma(t)) \pi^{\beta}(\sigma(t)), \quad t \geq t_{2} \geq t_{1} .
\end{aligned}
$$

Integrating (2.17) from $t_{2}$ to $t$, we have

$$
-r(t)\left(z^{\prime}(t)\right)^{\alpha} \geq C_{1}^{\beta} \int_{t_{2}}^{t} q(s) Q_{2}^{\beta}(\sigma(s)) \pi^{\beta}(\sigma(s)) d s,
$$

that is,

$$
-z^{\prime}(t) \geq \frac{C_{1}^{\beta / \alpha}}{r^{1 / \alpha}(t)}\left(\int_{t_{2}}^{t} q(s) Q_{2}^{\beta}(\sigma(s)) \pi^{\beta}(\sigma(s)) d s\right)^{\frac{1}{\alpha}}
$$

Integrating the above inequality from $t_{2}$ to $t$, we get

$$
z(t) \leq z\left(t_{2}\right)-\int_{t_{2}}^{t} \frac{C_{1}^{\beta / \alpha}}{r^{1 / \alpha}(u)}\left(\int_{t_{2}}^{u} q(s) Q_{2}^{\beta}(\sigma(s)) \pi^{\beta}(\sigma(s)) d s\right)^{\frac{1}{\alpha}} d u \rightarrow-\infty
$$

which contradicts the condition (2.16). The proof is complete.

Theorem 2.7 Let $\alpha \leq \beta$. Assume that (1.3), (2.6) and

$$
\int_{t_{1}}^{t} q(s) Q_{2}^{\beta}(\sigma(s)) \pi^{\beta}(\sigma(s)) d s=\infty
$$

hold. If

$$
\limsup _{t \rightarrow \infty} \pi^{\beta}(\sigma(t)) \int_{t_{1}}^{t} q(s) Q_{2}^{\beta}(\sigma(s)) d s>1 \quad \text { when } \alpha=\beta
$$

and

$$
\limsup _{t \rightarrow \infty} \pi^{\beta}(\sigma(t)) \int_{t_{1}}^{t} q(s) Q_{2}^{\beta}(\sigma(s)) d s>0 \quad \text { when } \alpha<\beta
$$

then Eq. (1.1) is oscillatory. 
Proof Suppose that $x(t)$ is a positive solution of equation (1.1) on $\left[t_{1}, \infty\right)$. From Lemma 2.4, we see that $z(t)$ satisfies Case 2 for $t \geq t_{1}$. Combining (2.18) and (2.19), we have

$$
\lim _{t \rightarrow \infty}\left(-r(t)\left(z^{\prime}(t)\right)^{\alpha}\right)=\infty
$$

Integrating (1.1) from $t_{1}$ to $t$ and using (2.4) and the fact that $z(t)$ is nonincreasing, we get

$$
\begin{aligned}
-r(t)\left(z^{\prime}(t)\right)^{\alpha} & =-r\left(t_{1}\right)\left(z^{\prime}\left(t_{1}\right)\right)^{\alpha}+\int_{t_{1}}^{t} q(s) x^{\beta}(\sigma(s)) d s \\
& \geq z^{\beta}(\sigma(t)) \int_{t_{1}}^{t} q(s) Q_{2}^{\beta}(\sigma(s)) d s .
\end{aligned}
$$

Noting (2.3) and $\sigma(t) \geq t$, we obtain

$$
\begin{aligned}
W(t) & :=-r(t)\left(z^{\prime}(t)\right)^{\alpha} \geq-r^{\frac{\beta}{\alpha}}(\sigma(t))\left(z^{\prime}(\sigma(t))\right)^{\beta} \pi^{\beta}(\sigma(t)) \int_{t_{1}}^{t} q(s) Q_{2}^{\beta}(\sigma(s)) d s \\
& =\left(-r(\sigma(t))\left(z^{\prime}(\sigma(t))\right)^{\alpha}\right)^{\frac{\beta}{\alpha}} \pi^{\beta}(\sigma(t)) \int_{t_{1}}^{t} q(s) Q_{2}^{\beta}(\sigma(s)) d s \\
& \geq\left(-r(t)\left(z^{\prime}(t)\right)^{\alpha}\right)^{\frac{\beta}{\alpha}} \pi^{\beta}(\sigma(t)) \int_{t_{1}}^{t} q(s) Q_{2}^{\beta}(\sigma(s)) d s \\
& =W^{\frac{\beta}{\alpha}}(t) \pi^{\beta}(\sigma(t)) \int_{t_{1}}^{t} q(s) Q_{2}^{\beta}(\sigma(s)) d s .
\end{aligned}
$$

Hence,

$$
W^{1-\frac{\beta}{\alpha}}(t) \geq \pi^{\beta}(\sigma(t)) \int_{t_{1}}^{t} q(s) Q_{2}^{\beta}(\sigma(s)) d s .
$$

Taking lim sup of both sides of the above inequality as $t \rightarrow \infty$, we arrive at a contradiction to (2.20) when $\alpha=\beta$ and (2.21) when $\alpha<\beta$. The proof is complete.

By attaching a condition, the dependence on the initial constant $t_{1}$ can be easily eliminated.

Corollary 2.8 Let $\alpha \leq \beta$. Assume that (1.3), (2.6), (2.9) and (2.19) hold. If

$$
\limsup _{t \rightarrow \infty} \pi^{\beta}(\sigma(t)) \int_{t_{0}}^{t} q(s) Q_{2}^{\beta}(\sigma(s)) d s>1 \quad \text { when } \alpha=\beta
$$

and

$$
\limsup _{t \rightarrow \infty} \pi^{\beta}(\sigma(t)) \int_{t_{0}}^{t} q(s) Q_{2}^{\beta}(\sigma(s)) d s>0 \quad \text { when } \alpha<\beta,
$$

then Eq. (1.1) is oscillatory.

Proof As in the proof of Theorem 2.7, we conclude that (2.23) holds. In view of (2.10), then there exists $t_{2}>t_{1}$ such that

$$
-r(t)\left(z^{\prime}(t)\right)^{\alpha}-z^{\beta}(\sigma(t)) \int_{t_{0}}^{t} q(s) Q_{2}^{\beta}(\sigma(s)) d s>0 .
$$


It is clear that

$$
\begin{aligned}
-r(t)\left(z^{\prime}(t)\right)^{\alpha} & \geq-r\left(t_{1}\right)\left(z^{\prime}\left(t_{1}\right)\right)^{\alpha}+\int_{t_{0}}^{t} q(s) x^{\beta}(\sigma(s)) d s-\int_{t_{0}}^{t_{1}} q(s) x^{\beta}(\sigma(s)) d s \\
& \geq z^{\beta}(\sigma(t)) \int_{t_{0}}^{t} q(s) Q_{2}^{\beta}(\sigma(s)) d s, \quad t>t_{1} .
\end{aligned}
$$

The rest of the proof is similar to that of Theorem 2.7 and hence we omit it.

In order to prove a main theorem of this paper, we review an auxiliary result obtained by Wu et al. [22, Lemma 2.3].

Lemma 2.9 Let $\varphi(u)=A u-B(u-C)^{(\alpha+1) / \alpha}$ where $\alpha>0$ is a quotient of two odd positive integers, $A$ and $C \in \mathbb{R}$, and $B>0$. Then $\varphi(u)$ attains its maximum value on $u^{*}=C+\left(\frac{A \alpha}{B(\alpha+1)}\right)^{\alpha}$, and

$$
\max _{u \in \mathbb{R}} \varphi(u)=\varphi\left(u^{*}\right)=A C+\frac{\alpha^{\alpha}}{(\alpha+1)^{\alpha+1}} \frac{A^{\alpha+1}}{B^{\alpha}} .
$$

The proof of the above lemma is simple and can be obtained directly by the change of the variable. We omit it.

Theorem 2.10 Let $\alpha>\beta$. Assume that (1.3) and (2.6) hold. If there exists a function $\rho \in$ $C^{1}\left(\left[t_{0}, \infty\right),(0, \infty)\right)$ such that

$$
\limsup _{t \rightarrow \infty}\left(\frac{\pi^{\alpha}(t)}{\rho(t)} \int_{t_{2}}^{t} \rho(s) q(s) Q_{2}^{\beta}(\sigma(s))\left(\frac{\pi(\sigma(s))}{\pi(s)}\right)^{\beta}-\frac{\alpha^{\alpha} C_{4}^{\alpha}\left(\rho^{\prime}(s)\right)^{\alpha+1} r(s)}{(\alpha+1)^{\alpha+1} \beta^{\alpha} \rho^{\alpha}(s)} d s\right)>1,
$$

for any positive constants $C_{4}>0$ and $t_{2} \geq t_{0}$, then Eq. (1.1) is oscillatory.

Proof Suppose that $x(t)$ is a positive solution of equation (1.1) on $\left[t_{1}, \infty\right)$. From Lemma 2.4, we see that $z(t)$ satisfies Case 2 for $t \geq t_{1}$. Define the generalized Riccati substitution $w(t)$ by

$$
w(t):=\rho(t)\left(\frac{r(t)\left(z^{\prime}(t)\right)^{\alpha}}{z^{\beta}(t)}+\frac{1}{\pi^{\alpha}(t)}\right), \quad t \geq t_{1} .
$$

By virtue of (2.3), we have $w(t) \geq 0$ for $t \geq t_{1}$. Differentiating on both sides of (2.27), we obtain

$$
\begin{aligned}
w^{\prime}(t) & =\frac{\rho^{\prime}(t)}{\rho(t)} w(t)+\rho(t) \frac{\left(r(t)\left(z^{\prime}(t)\right)^{\alpha}\right)^{\prime}}{z^{\beta}(t)}-\frac{\beta \rho(t) r(t)\left(z^{\prime}(t)\right)^{\alpha} z^{\prime}(t)}{z^{\beta+1}(t)}+\frac{\alpha \rho(t)}{r^{1 / \alpha}(t) \pi^{\alpha}(t)} \\
& \leq \frac{\rho^{\prime}(t)}{\rho(t)} w(t)+\rho(t) \frac{\left(r(t)\left(z^{\prime}(t)\right)^{\alpha}\right)^{\prime}}{z^{\beta}(t)}-\frac{\beta\left(w(t)-\frac{\rho(t)}{\pi^{\alpha}(t)}\right)^{\frac{\alpha+1}{\alpha}}}{C_{4}\left(\rho(t)(r(t))^{1 / \alpha}\right.}+\frac{\alpha \rho(t)}{r^{1 / \alpha}(t) \pi^{\alpha}(t)}
\end{aligned}
$$

where $C_{4}>0$ is a constant and such that $z^{1-\frac{\beta}{\alpha}}(t) \leq C_{4}$ for $t \geq t_{1}$. 
Combining (1.1), $\sigma(t) \geq t$ and Lemma 2.3, we have

$$
\left(r(t)\left(z^{\prime}(t)\right)^{\alpha}\right)^{\prime} \leq-q(t) Q_{2}^{\beta}(\sigma(t)) z^{\beta}(\sigma(t)) \leq-q(t) Q_{2}^{\beta}(\sigma(t))\left(\frac{\pi(\sigma(t))}{\pi(t)}\right)^{\beta} z^{\beta}(\sigma(t))
$$

for $t>t_{2}$, where $t_{2} \in\left[t_{1}, \infty\right)$ is large enough. Substituting (2.29) into (2.28), it follows that

$$
\begin{aligned}
w^{\prime}(t) \leq & -\rho(t) q(t) Q_{2}^{\beta}(\sigma(t))\left(\frac{\pi(\sigma(t))}{\pi(t)}\right)^{\beta}+\frac{\rho^{\prime}(t)}{\rho(t)} w(t) \\
& -\frac{\beta}{C_{4}\left(\rho(t)(r(t))^{1 / \alpha}\right.}\left(w(t)-\frac{\rho(t)}{\pi^{\alpha}(t)}\right)^{\frac{\alpha+1}{\alpha}}+\frac{\alpha \rho(t)}{r^{1 / \alpha}(t) \pi^{\alpha}(t)} .
\end{aligned}
$$

Using (2.25) with

$$
A:=\frac{\rho^{\prime}(t)}{\rho(t)}, \quad B:=\frac{\beta}{C_{4}\left(\rho(t)(r(t))^{1 / \alpha}\right.}, \quad C:=\frac{\rho(t)}{\pi^{\alpha}(t)},
$$

we obtain

$$
\begin{aligned}
w^{\prime}(t) \leq & -\rho(t) q(t) Q_{2}^{\beta}(\sigma(t))\left(\frac{\pi(\sigma(t))}{\pi(t)}\right)^{\beta}+\frac{\rho^{\prime}(t)}{\pi^{\alpha}(t)} \\
& +\frac{\alpha^{\alpha} C_{4}^{\alpha}\left(\rho^{\prime}(t)\right)^{\alpha+1} r(t)}{(\alpha+1)^{\alpha+1} \beta^{\alpha} \rho^{\alpha}(t)}+\frac{\alpha \rho(t)}{r^{1 / \alpha}(t) \pi^{\alpha}(t)} \\
\leq & -\rho(t) q(t) Q_{2}^{\beta}(\sigma(t))\left(\frac{\pi(\sigma(t))}{\pi(t)}\right)^{\beta}+\left(\frac{\rho(t)}{\pi^{\alpha}(t)}\right)^{\prime} .
\end{aligned}
$$

Integrating (2.31) from $t_{2}$ to $t$, we have

$$
\begin{aligned}
& \int_{t_{2}}^{t} \rho(s) q(s) Q_{2}^{\beta}(\sigma(s))\left(\frac{\pi(\sigma(s))}{\pi(s)}\right)^{\beta}-\frac{\alpha^{\alpha} C_{4}^{\alpha}\left(\rho^{\prime}(s)\right)^{\alpha+1} r(s)}{(\alpha+1)^{\alpha+1} \beta^{\alpha} \rho^{\alpha}(s)} d s-\frac{\rho(t)}{\pi^{\alpha}(t)}+\frac{\rho\left(t_{2}\right)}{\pi^{\alpha}\left(t_{2}\right)} \\
& \quad \leq w\left(t_{2}\right)-w(t) .
\end{aligned}
$$

In view of (2.27), we see that

$$
\begin{aligned}
& \int_{t_{2}}^{t} \rho(s) q(s) Q_{2}^{\beta}(\sigma(s))\left(\frac{\pi(\sigma(s))}{\pi(s)}\right)^{\beta}-\frac{\alpha^{\alpha} C_{4}^{\alpha}\left(\rho^{\prime}(s)\right)^{\alpha+1} r(s)}{(\alpha+1)^{\alpha+1} \beta^{\alpha} \rho^{\alpha}(s)} d s \\
& \quad \leq \rho\left(t_{2}\right) \frac{r\left(t_{2}\right)\left(z^{\prime}\left(t_{2}\right)\right)^{\alpha}}{z^{\beta}\left(t_{2}\right)}-\rho(t) \frac{r(t)\left(z^{\prime}(t)\right)^{\alpha}}{z^{\beta}(t)} .
\end{aligned}
$$

On the other hand, from (2.3), we have

$$
-\frac{\rho(t)}{\pi^{\alpha}(t)} \leq \rho(t) \frac{r(t)\left(z^{\prime}(t)\right)^{\alpha}}{z^{\beta}(t)} \leq 0
$$

Substituting (2.33) into (2.32), we obtain

$$
\int_{t_{2}}^{t} q(s) Q_{2}^{\beta}(\sigma(s))\left(\frac{\pi(\sigma(s))}{\pi(s)}\right)^{\beta}-\frac{\alpha^{\alpha} C_{4}^{\alpha}\left(\rho^{\prime}(s)\right)^{\alpha+1} r(s)}{(\alpha+1)^{\alpha+1} \beta^{\alpha} \rho^{\alpha}(s)} d s \leq \frac{\rho(t)}{\pi^{\alpha}(t)} .
$$


Multiplying both sides of (2.34) by $\frac{\pi^{\alpha}(t)}{\rho(t)}$ and taking lim sup on both sides of the resulting inequality as $t \rightarrow \infty$, we obtain a contradiction to (2.26). The proof is complete.

Since $\rho(t)$ can be taken appropriately, Theorem 2.10 is more flexible in studying the oscillation of (1.1). When $\rho(t)=\pi^{\alpha}(t), \rho(t)=\pi^{\beta}(t), \rho(t)=1$, respectively, the following results are obtained.

Corollary 2.11 Assume that $\alpha>\beta$, (1.3) and (2.6) hold. If

$$
\limsup _{t \rightarrow \infty}\left(\int_{t_{2}}^{t} q(s) Q_{2}^{\beta}(\sigma(s)) \pi^{\beta}(\sigma(s)) \pi^{\alpha-\beta}(s)-\frac{C_{4}^{\alpha} \alpha^{2 \alpha+1}}{\beta^{\alpha}(\alpha+1)^{\alpha+1} r^{1 / \alpha}(s) \pi^{\alpha}(s)} d s\right)>1,
$$

for any positive constants $C_{4}>0$ and $t_{2} \geq t_{0}$, then Eq. (1.1) is oscillatory.

Corollary 2.12 Assume that $\alpha>\beta$, (1.3) and (2.6) hold. If

$$
\limsup _{t \rightarrow \infty}\left(\pi^{\alpha-\beta}(t) \int_{t_{2}}^{t} q(s) Q_{2}^{\beta}(\sigma(s)) \pi^{\beta}(\sigma(s))-\frac{C_{4}^{\alpha} \alpha^{\alpha} \beta}{(\alpha+1)^{\alpha+1} r^{1 / \alpha}(s) \pi^{\alpha-\beta+1}(s)} d s\right)>1,
$$

for any positive constants $C_{4}>0$ and $t_{2} \geq t_{0}$, then Eq. (1.1) is oscillatory.

Corollary 2.13 Assume that $\alpha \geq \beta$, (1.3) and (2.6) hold. If

$$
\limsup _{t \rightarrow \infty}\left(\pi^{\alpha}(t) \int_{t_{2}}^{t} q(s) Q_{2}^{\beta}(\sigma(s))\left(\frac{\pi(\sigma(s))}{\pi(s)}\right)^{\beta} d s\right)>1,
$$

for any $t_{2} \geq t_{0}$, then Eq. (1.1) is oscillatory.

Remark When $\alpha=\beta$, we can choose $C_{4}=1$ in Theorem 2.10, Corollary 2.11, Corollary 2.12 , respectively.

Lemma 2.14 Let $\alpha \leq \beta$. Assume that (1.3) and (2.6) hold. Suppose that equation (1.1) has a positive solution $x(t)$ on $\left[t_{1}, \infty\right)$ and that $\gamma$ and $\delta$ are constants satisfying

$$
\begin{aligned}
& 0 \leq \gamma+\delta<1, \\
& 0 \leq \gamma \leq L q(t) Q_{2}^{\beta}(\sigma(t)) \pi^{\beta}(\sigma(t)) \pi(t) r^{\frac{1}{\alpha}}(t),
\end{aligned}
$$

where $L>0$ is a constant and such that $\left(r(t)\left(z^{\prime}(t)\right)^{\alpha}\right)^{\frac{\beta-\alpha}{\alpha}} \geq L$ for $t \geq t_{1}$, and

$$
0 \leq \delta \leq m_{1} \pi(\sigma(t))\left(\int_{t_{1}}^{t} q(s) Q_{1}^{\beta}(\sigma(s)) d s\right)^{\frac{1}{\alpha}},
$$

where $m_{1}>0$ is a constant and such that $z^{\beta-\alpha}(\sigma(t)) \geq m_{1}$. Then there exists $t_{*} \in\left[t_{1}, \infty\right)$ such that

$$
\frac{z}{\pi^{1-\gamma}}
$$


is nondecreasing and

$$
\frac{z}{\pi^{\delta}}
$$

is nonincreasing on $\left[t_{*}, \infty\right)$.

Proof From Lemma 2.4, we see that $z(t)$ satisfies Case 2. Using (1.1), (2.3), (2.4) and (2.36), we obtain

$$
\begin{aligned}
(-r(t) & \left.\left(z^{\prime}(t)\right)^{\alpha} \pi^{\gamma}(t)\right)^{\prime} \\
= & -\left(r(t)\left(z^{\prime}(t)\right)^{\alpha}\right)^{\prime} \pi^{\gamma}(t)+\gamma r(t)\left(z^{\prime}(t)\right)^{\alpha} \frac{\pi^{\gamma-1}(t)}{r^{1 / \alpha}(t)} \\
= & q(t) x^{\beta}(\sigma(t)) \pi^{\gamma}(t)+\gamma r(t)\left(z^{\prime}(t)\right)^{\alpha} \frac{\pi^{\gamma-1}(t)}{r^{1 / \alpha}(t)} \\
\geq & -q(t) Q_{2}^{\beta}(\sigma(t)) \pi^{\gamma}(t) r^{\beta / \alpha}(\sigma(t))\left(z^{\prime}(\sigma(t))\right)^{\beta} \pi^{\beta}(\sigma(t)) \\
& +\gamma r(t)\left(z^{\prime}(t)\right)^{\alpha} \frac{\pi^{\gamma-1}(t)}{r^{1 / \alpha}(t)} \\
\geq & -q(t) Q_{2}^{\beta}(\sigma(t)) \pi^{\gamma}(t) r(t)\left(z^{\prime}(t)\right)^{\alpha}\left(r(\sigma(t))\left(z^{\prime}(\sigma(t))\right)^{\alpha}\right)^{\frac{\beta-\alpha}{\alpha}} \pi^{\beta}(\sigma(t)) \\
& +\gamma r(t)\left(z^{\prime}(t)\right)^{\alpha} \frac{\pi^{\gamma-1}(t)}{r^{1 / \alpha}(t)} \\
\geq & -q(t) Q_{2}^{\beta}(\sigma(t)) \pi^{\gamma}(t) r(t)\left(z^{\prime}(t)\right)^{\alpha} L \pi^{\beta}(\sigma(t)) \\
& +\gamma r(t)\left(z^{\prime}(t)\right)^{\alpha} \frac{\pi^{\gamma-1}(t)}{r^{1 / \alpha}(t)} \\
= & -r(t)\left(z^{\prime}(t)\right)^{\alpha} \pi^{\gamma}(t)\left[L q(t) Q_{2}^{\beta}(\sigma(t)) \pi^{\beta}(\sigma(t))-\frac{\gamma}{\pi(t) r^{1 / \alpha}(t)}\right] \geq 0,
\end{aligned}
$$

where $L>0$ is a constant and such that $\left(r(t)\left(z^{\prime}(t)\right)^{\alpha}\right)^{\frac{\beta-\alpha}{\alpha}} \geq L$ for $t \geq t_{1}$. Thus, $-r(t)\left(z^{\prime}(t)\right)^{\alpha} \times$ $\pi^{\gamma}(t)$ is nondecreasing eventually, that is, there exists a $t_{2} \in\left[t_{1}, \infty\right)$ such that $-r(t)\left(z^{\prime}(t)\right)^{\alpha} \times$ $\pi^{\gamma}(t)$ is nondecreasing for $t \geq t_{2}$. So, we have

$$
\begin{aligned}
z(t) & \geq-\int_{t}^{\infty} \frac{r^{1 / \alpha}(s) \pi^{\gamma}(s)}{r^{1 / \alpha}(s) \pi^{\gamma}(s)} z^{\prime}(s) d s \\
& \geq-r^{1 / \alpha}(t) z^{\prime}(t) \pi^{\gamma}(t) \int_{t}^{\infty} \frac{1}{r^{1 / \alpha}(s) \pi^{\gamma}(s)} d s .
\end{aligned}
$$

In view of

$$
\int_{t}^{\infty} \frac{1}{r^{1 / \alpha}(s) \pi^{\gamma}(s)} d s=\frac{\pi^{1-\gamma}(t)}{1-\gamma}
$$

we get

$$
z(t) \geq-r^{1 / \alpha}(t) z^{\prime}(t) \frac{\pi(t)}{1-\gamma}
$$


Hence,

$$
\left(\frac{z(t)}{\pi^{1-\gamma}(t)}\right)^{\prime}=\frac{r^{1 / \alpha}(t) z^{\prime}(t) \frac{\pi(t)}{1-\gamma}+z(t)}{(1-\gamma) r^{1 / \alpha}(t) \pi^{2-\gamma}(t)} \geq 0
$$

that is, $\frac{z(t)}{\pi^{1-\gamma}(t)}$ is nondecreasing.

Next, we prove that $\frac{z}{\pi^{\delta}}$ is nonincreasing. Proceeding as in the proof of Theorem 2.5, we obtain (2.15), that is,

$$
z(t) \leq-\frac{1}{m_{1}} r^{1 / \alpha}(t) z^{\prime}(t) \frac{\pi(t)}{\pi(\sigma(t))}\left(\int_{t_{0}}^{t} q(s) Q_{2}^{\beta}(\sigma(s)) d s\right)^{-\frac{1}{\alpha}}
$$

where $m_{1}>0$ is a constant and such that $z^{\beta-\alpha}(\sigma(t)) \geq m_{1}$. On the other hand, we see

$$
\left(\frac{z(t)}{\pi^{\delta}(t)}\right)^{\prime}=\frac{z^{\prime}(t)}{\pi^{\delta}(t)}+\frac{\delta z(t)}{\pi^{\delta+1}(t) r^{1 / \alpha}(t)}
$$

Using the inequality (2.42), we obtain

$$
\begin{aligned}
\left(\frac{z(t)}{\pi^{\delta}(t)}\right)^{\prime} & \leq \frac{z^{\prime}(t)}{\pi^{\delta}(t)}-\frac{\delta z^{\prime}(t)}{m_{1} \pi^{\delta}(t) \pi(\sigma(t))}\left(\int_{t_{0}}^{t} q(s) Q_{2}^{\beta}(\sigma(s)) d s\right)^{-\frac{1}{\alpha}} \\
& =\frac{z^{\prime}(t)}{\pi^{\delta}(t)}\left(1-\frac{\delta}{m_{1} \pi(\sigma(t))}\left(\int_{t_{0}}^{t} q(s) Q_{2}^{\beta}(\sigma(s)) d s\right)^{-\frac{1}{\alpha}}\right) \leq 0
\end{aligned}
$$

Thus, $\frac{z(t)}{\pi^{\delta}(t)}$ is nonincreasing. The proof is complete.

Theorem 2.15 Let $\alpha \leq \beta$. Assume that (1.3) and (2.6) hold. Suppose that $\gamma$ and $\delta$ are constants satisfying (2.35)-(2.37). Also, there exists a constant $M>0$ such that $\int_{t_{0}}^{t} q(t) d s \leq$ $M$ for all $t \geq t_{0}$. If

$$
\begin{aligned}
& \limsup _{t \rightarrow \infty} \pi^{\gamma}(t) \pi^{1-\gamma-\delta}(\sigma(t)) \int_{t_{1}}^{t} \pi^{\delta \beta}(\sigma(s)) q(s) Q_{2}^{\beta}(\sigma(s)) d s>(1-\gamma)^{\beta} \\
& \text { when } \alpha=\beta
\end{aligned}
$$

and

$$
\begin{aligned}
& \limsup _{t \rightarrow \infty} \pi^{\gamma}(t) \pi^{1-\gamma-\delta}(\sigma(t)) \int_{t_{1}}^{t} \pi^{\delta \beta}(\sigma(s)) q(s) Q_{2}^{\beta}(\sigma(s)) d s=\infty \\
& \text { when } \alpha<\beta
\end{aligned}
$$

for any $t_{1} \geq t_{0}$, then Eq. (1.1) is oscillatory.

Proof Suppose that $x(t)$ is a positive solution of Eq. (1.1) on $\left[t_{1}, \infty\right)$. From Lemma 2.4, we see that $z$ satisfies Case 2 for $t \geq t_{1}$. 
Integrating from $t_{1}$ to $t$ and combining Lemma 2.14, we have

$$
\begin{aligned}
W(t) & :=-r(t)\left(z^{\prime}(t)\right)^{\alpha}=-r\left(t_{1}\right)\left(z^{\prime}\left(t_{1}\right)\right)^{\alpha}+\int_{t_{1}}^{t} q(s) x^{\beta}(\sigma(s)) d s \\
& \geq \int_{t_{1}}^{t}\left(\frac{z(\sigma(s))}{\pi^{\delta}(\sigma(s))}\right)^{\beta} \pi^{\delta \beta}(\sigma(s)) q(s) Q_{2}^{\beta}(\sigma(s)) d s \\
& \geq\left(\frac{z(\sigma(t))}{\pi^{\delta}(\sigma(t))}\right)^{\beta} \int_{t_{1}}^{t} \pi^{\delta \beta}(\sigma(s)) q(s) Q_{2}^{\beta}(\sigma(s)) d s \\
& \geq\left(\frac{z(\sigma(t)) \pi^{1-\gamma}(\sigma(t))}{\pi^{\delta}(\sigma(t)) \pi^{1-\gamma}(\sigma(t))}\right)^{\beta} \int_{t_{1}}^{t} \pi^{\delta \beta}(\sigma(s)) q(s) Q_{2}^{\beta}(\sigma(s)) d s \\
& \geq\left(\frac{z(t) \pi^{1-\gamma-\delta}(\sigma(t))}{\pi^{1-\gamma}(t)}\right)^{\beta} \int_{t_{1}}^{t} \pi^{\delta \beta}(\sigma(s)) q(s) Q_{2}^{\beta}(\sigma(s)) d s .
\end{aligned}
$$

Clearly, one can see that the function $W(t)$ is bounded due to Eq. (1.1) and condition $\int_{t_{0}}^{t} q(t) d s \leq M$. Using (2.41) in the above inequality, we obtain

$$
W(t) \geq W^{\frac{\beta}{\alpha}}(t)\left(\frac{\pi^{\gamma}(t) \pi^{1-\gamma-\delta}(\sigma(t))}{1-\gamma}\right)^{\beta} \int_{t_{1}}^{t} \pi^{\delta \beta}(\sigma(s)) q(s) Q_{2}^{\beta}(\sigma(s)) d s
$$

that is,

$$
W^{1-\frac{\beta}{\alpha}}(t)(t) \geq\left(\frac{\pi^{\gamma}(t) \pi^{1-\gamma-\delta}(\sigma(t))}{1-\gamma}\right)^{\beta} \int_{t_{1}}^{t} \pi^{\delta \beta}(\sigma(s)) q(s) Q_{2}^{\beta}(\sigma(s)) d s
$$

Taking lim sup on both sides of this inequality, we arrive at a contradiction to (2.44) when $\alpha=\beta$ and (2.45) when $\alpha<\beta$. The proof is complete.

\section{Examples}

In this section, we present two examples to illustrate our main results.

Example 3.1 Consider the following second order differential equation:

$$
\left[t^{4}\left(x(t)+\frac{1}{9} x\left(\frac{t}{2}\right)\right)^{\prime}\right]^{\prime}+t^{6} x^{\frac{5}{3}}(3 t)=0, \quad t \geq 1 .
$$

Clearly,

$$
\begin{array}{llll}
r(t)=t^{4}, & p_{1}(t)=\frac{1}{9}, & p_{2}(t)=0, & q(t)=t^{6}, \\
\tau(t)=\frac{t}{2}, & \sigma(t)=3 t, & \alpha=1, & \beta=\frac{5}{3} .
\end{array}
$$

By $\pi(t)=\int_{t}^{\infty} r^{-\frac{1}{\alpha}}(s) d s$, we have $\pi(t)=\frac{1}{3} t^{-3}$, and condition (1.3) holds. Notice that $\frac{\pi(\tau(\sigma(t)))}{\pi(\sigma(t))}=\frac{\frac{1}{3}\left(\frac{3 t}{2}\right)^{-3}}{\frac{1}{3}(3 t)^{-3}}=8$, and

$$
Q_{1}^{\beta}(t)=\left(1-p_{1}(\sigma(t))-p_{2}(\sigma(t)) \frac{R(\lambda(\sigma(t)))}{R(\sigma(t))}\right)^{\beta}=\left(\frac{8}{9}\right)^{\frac{5}{3}},
$$




$$
Q_{2}^{\beta}(t)=\left(1-p_{1}(\sigma(t)) \frac{\pi(\tau(\sigma(t)))}{\pi(\sigma(t))}-p_{2}(\sigma(t))\right)^{\beta}=\left(\frac{1}{9}\right)^{\frac{5}{3}} .
$$

Letting $t_{1}=t_{0}=1$, we have

$$
\limsup _{t \rightarrow \infty} \int_{1}^{t} s^{6}\left(\frac{8}{9}\right)^{\frac{5}{3}} d s=\infty
$$

and condition (2.6) is satisfied. To verify conditions (2.19) and (2.21), we find

$$
\int_{t_{1}}^{t} q(s) Q_{2}^{\beta}(\sigma(s)) \pi^{\beta}(\sigma(s)) d s=\int_{1}^{t} s^{6}\left(\frac{1}{9}\right)^{\frac{5}{3}}\left(\frac{1}{3}\right)^{\frac{5}{3}}(3 s)^{-5} d s=\infty
$$

and

$$
\limsup _{t \rightarrow \infty} \pi^{\beta}(\sigma(t)) \int_{t_{1}}^{t} q(s) Q_{2}^{\beta}(\sigma(s)) d s=\limsup _{t \rightarrow \infty}\left(\frac{1}{3}\right)^{\frac{5}{3}}(3 t)^{-5} \int_{1}^{t} s^{6}\left(\frac{1}{9}\right)^{\frac{5}{3}} d s>0,
$$

which show that (2.19) and (2.21) hold. Hence, by Theorem 2.7, Eq. (3.1) is oscillatory.

Example 3.2 Consider the following second order differential equation:

$$
\left[t^{4}\left(x(t)+\frac{1}{9} x\left(\frac{t}{2}\right)\right)^{\prime}\right]^{\prime}+t^{6} x^{\frac{1}{3}}(3 t)=0, \quad t \geq 1 .
$$

It is easy to find that

$$
\begin{array}{ll}
r(t)=t^{4}, & p_{1}(t)=\frac{1}{9}, \quad p_{2}(t)=0, \quad q(t)=t^{6}, \\
\tau(t)=\frac{t}{2}, & \sigma(t)=3 t, \quad \alpha=1, \quad \beta=\frac{1}{3} .
\end{array}
$$

From $\pi(t)=\int_{t}^{\infty} r^{-\frac{1}{\alpha}}(s) d s$, we have $\pi(t)=\frac{1}{3} t^{-3}$ and condition (1.3) holds. In view of $\frac{\pi(\tau(\sigma(t)))}{\pi(\sigma(t))}=\frac{\frac{1}{3}\left(\frac{3 t}{2}\right)^{-3}}{\frac{1}{3}(3 t)^{-3}}=8$, we obtain

$$
\begin{aligned}
& Q_{1}^{\beta}(t)=\left(1-p_{1}(\sigma(t))-p_{2}(\sigma(t)) \frac{R(\lambda(\sigma(t)))}{R(\sigma(t))}\right)^{\beta}=\left(\frac{8}{9}\right)^{\frac{1}{3}}, \\
& Q_{2}^{\beta}(t)=\left(1-p_{1}(\sigma(t)) \frac{\pi(\tau(\sigma(t)))}{\pi(\sigma(t))}-p_{2}(\sigma(t))\right)^{\beta}=\left(\frac{1}{9}\right)^{\frac{1}{3}} .
\end{aligned}
$$

Letting $t_{1}=t_{0}=1$, we see that

$$
\limsup _{t \rightarrow \infty} \int_{1}^{t} s^{6}\left(\frac{8}{9}\right)^{\frac{1}{3}} d s=\infty
$$


and condition (2.6) is satisfied. Setting $\rho(t)=1$, we have

$$
\begin{gathered}
\limsup _{t \rightarrow \infty}\left(\pi^{\alpha}(t) \int_{t_{2}}^{t} q(s) Q_{2}^{\beta}(\sigma(s))\left(\frac{\pi(\sigma(s))}{\pi(s)}\right)^{\beta} d s\right) \\
\quad=\limsup _{t \rightarrow \infty} \frac{1}{3} t^{-3} \int_{1}^{t} s^{6}\left(\frac{1}{9}\right)^{\frac{1}{3}} \frac{1}{3} d s>1 .
\end{gathered}
$$

Now, all conditions of Corollary 2.13 hold. Hence, Eq. (3.2) is oscillatory.

\section{Conclusions}

In this paper, we have obtained several new oscillation criteria for a second order nonlinear advanced differential equation with mixed neutral terms. Our results improve and complement some well-known results which were published recently in the literature. Two examples are given to illustrate the efficiency of our results. We believe that the proof method and the obtained results may be generalized to the differential equations, such as those in $[11,13,18]$.

\section{Acknowledgements}

The authors thank the editor and anonymous referees for their valuable suggestions and comments, which improved the presentation of this paper.

\section{Funding}

This work was supported by the National Natural Science Foundation of China (No. 11671227) and Natural Science Foundation of Shandong Province of China (No. ZR2019MA067).

\section{Availability of data and materials}

All data are fully available without restriction.

\section{Competing interests}

The authors declare that they have no competing interests.

\section{Authors' contributions}

The two authors contributed equally to this work. They read and approved the final version of the manuscript.

\section{Publisher's Note}

Springer Nature remains neutral with regard to jurisdictional claims in published maps and institutional affiliations.

Received: 12 July 2019 Accepted: 24 October 2019 Published online: 14 November 2019

\section{References}

1. Agarwal, R.P., Bohner, M., Li, T., Zhang, C.: Oscillation of second-order Emden-Fowler neutral delay differential equations. Ann. Mat. Pura Appl. (4) 193, 1861-1875 (2014)

2. Agarwal, R.P., Bohner, M., Li, T., Zhang, C.: Even-order half-linear advanced differential equations: improved criteria in oscillatory and asymptotic properties. Appl. Math. Comput. 266, 481-490 (2015)

3. Agarwal, R.P., Bohner, M., Li, W.: Nonoscillation and Oscillation: Theory for Functional Differential Equations. Monographs and Textbooks in Pure and Applied Mathematics, vol. 267. Dekker, New York (2004)

4. Agarwal, R.P., Grace, S.R., O'Regan, D.: Oscillation Theory for Second Order Linear, Half-Linear, Superlinear and Sublinear Dynamic Equations. Kluwer Academic, Dordrecht (2002)

5. Agarwal, R.P., Grace, S.R., O'Regan, D.: Oscillation Theory for Second Order Dynamic Equations. Series in Mathematical Analysis and Applications. Taylor \& Francis, London (2003)

6. Agarwal, R.P., Grace, S.R., O'Regan, D.: Oscillation Theory for Difference and Functional Differential Equations. Springer, Berlin (2013)

7. Agarwal, R.P., Zhang, C., Li, T.: New Kamenev-type oscillation criteria for second-order nonlinear advanced dynamic equations. Appl. Math. Comput. 225, 822-828 (2013)

8. Agarwal, R.P., Zhang, C., Li, T.: Some remarks on oscillation of second order neutral differential equations. Appl. Math. Comput. 274, 178-181 (2016)

9. Baculíková, B.: Oscillatory behavior of the second order functional differential equations. Appl. Math. Lett. 72, 35-41 (2017)

10. Chatzarakis, G.E., Džurina, J., Jadlovská, I.: New oscillation criteria for second-order half-linear advanced differential equations. Appl. Math. Comput. 347, 404-416 (2019) 
11. Chatzarakis, G.E., Grace, S.R., Jadlovská, I., Li, T., Tunç, E.: Oscillation criteria for third-order Emden-Fowler differential equations with unbounded neutral coefficients. Complexity 2019, Article ID 5691758 (2019)

12. Džurina, J.: A comparison theorem for linear delay differential equations. Arch. Math. 135, 531-540 (2003)

13. Džurina, J., Grace, S.R., Jadlovská, I., Li, T.: Oscillation criteria for second-order Emden-Fowler delay differential equations with a sublinear neutral term. Math. Nachr. https://doi.org/10.1002/mana.201800196

14. Elsgolts, L.E.. Norkin, S.B.: Introduction to the Theory and Application of Differential Equations with Deviating Arguments. Academic Press, New York (1973)

15. Fite, W.B.: Properties of the solutions of certain functional-differential equations. Trans. Am. Math. Soc. 22, 311-319 (1921)

16. Grace, S.R., Džurina, J., Jadlovská, I., Li, T.: An improved approach for studying oscillation of second-order neutral delay differential equations. J. Inequal. Appl. 2018, 193 (2018)

17. Jadlovská, I.: Iterative oscillation results for second-order differential equations with advanced argument. Electron. J. Differ. Equ. 2017, 162 (2017)

18. Li, T., Rogovchenko, Y.V.: Asymptotic behavior of higher-order quasilinear neutral differential equations. Abstr. Appl. Anal. 2014, Article ID 395368 (2014)

19. Li, T., Rogovchenko, Y.V.: Oscillation of second-order neutral differential equations. Math. Nachr. 288, 1150-1162 (2015)

20. Li, T., Rogovchenko, Y.V.: Oscillation criteria for second-order superlinear Emden-Fowler neutral differential equations. Monatshefte Math. 184, 489-500 (2017)

21. Trench, W.F.: Canonical forms and principal systems for general disconjugate equations. Trans. Am. Math. Soc. 189 319-327 (1973)

22. Wu, H., Erbe, L., Peterson, A.: Oscillation of solution to second-order half-linear delay dynamic equations on time scales. Electron. J. Differ. Equ. 2016, 71 (2016)

23. Zhang, C., Agarwal, R.P., Bohner, M., Li, T.: Oscillation of second-order nonlinear neutral dynamic equations with noncanonical operators. Bull. Malays. Math. Sci. Soc. 38, 761-778 (2015)

\section{Submit your manuscript to a SpringerOpen ${ }^{\circ}$ journal and benefit from:}

- Convenient online submission

- Rigorous peer review

- Open access: articles freely available online

- High visibility within the field

- Retaining the copyright to your article

Submit your next manuscript at $\gg$ springeropen.com 